\title{
PIEZOELECTRICALLY ACTUATED MICROCANTILEVER FOR ACTUATED MIRROR ARRAY APPLICATION
}

\author{
Yongli Huang, Hong Zhang, and Eun Sok Kim \\ Department of Electrical Engineering \\ University of Hawaii at Manoa \\ Honolulu, Hawaii 96822 \\ Sang Gook Kim and Yong Bae Jeon \\ Daewoo Electronics Co. \\ Seoul, Korea 100-714
}

\begin{abstract}
For a projection display application, we have developed a piezoelectrically actuated array of cantilevers whose facets can accurately be controlled for a desired gray level. A $40 \times 40$ cantilever array with a pixel size of $100 \times 100$ $\mu \mathrm{m}^{2}$ has been fabricated, and a piezoelectric $\mathrm{ZnO}$ thin film (in spite of its relatively low $\mathrm{d}_{31}$ ) is successfully used to produce $0.116^{\circ} / \mathrm{V}$ vertical deflection of the cantilever. In the fabrication processing, we have discovered that many materials have their unique critical HF concentration below which there is no etching by vapor HF, and successfully used vapor $\mathrm{HF}$ to release cantilevers with a very high yield and a processing simplicity.
\end{abstract}

\section{INTRODUCTION}

In the digital mirror array [1] and the grating light valves [2] for a projection display application, individual mirror elements are deformed or tilted electrostatically. The control of mirror positions in those devices is basically bistable in that the mirror elements are tilted or deformed into either one of two stable positions. Consequently, the gray scale control inherently requires high speed digital circuitry. Moreover, since the mirror elements are constantly banged into the substrate during the operations, long-term reliability may suffer due to in-operation stiction.

On the other hand, a piezoelectric actuation of mirror elements allows us to have linear control of the mirror tilting, and the gray scale control becomes very simple. Also, the mirror elements formed by surface micromachining never have to touch the substrate during operation, and the in-operation stiction is not a concern. We have fabricated an array of piezolectrically actuated cantilevers for a projection display system illustrated in Fig. 1. In the system, the piezoelectrically actuated cantilevers are individually tilted for an image formation through a light modulation technique shown in Fig. 2.

With bulk piezo-ceramics (PLZT), a $200 \times 400$ array of mirror elements was demonstrated to show high light throughput efficiency [3].

A ferroelectric PZT film has large piezoelectric constant (e.g., d 31 ), and can advantageously be used to actuate microstructures. However, PZT thin film lacks a track record up to now. While sputter deposition of PZT requires very tight process control for repeatable quality of PZT films, sol-gel PZT typically has large residual stress. In contrast, a piezoelectric $\mathrm{ZnO}$ thin film has been extensively used in commercial SAW filters for TV's IF filters (tens of million devices per year), and has been proven to be reliable and reproducible (i.e., the $\mathrm{ZnO}$ properties are well controlled).

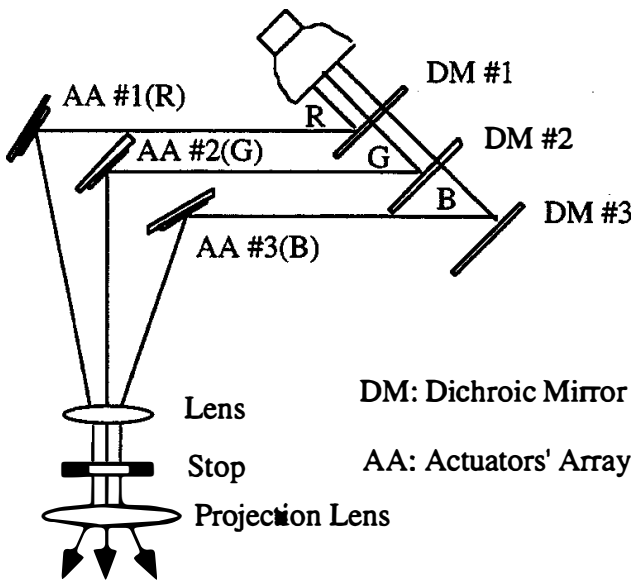

Figure 1. Conceptual picture of the projection display system using the piezoelectrically actuated mirror array.
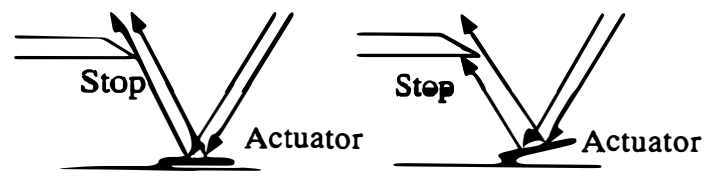

a) No Tilting (100\% passing) b) Tilting ( $50 \%$ passing) Figure 2. The light modulation technique with a knife-edge filter.

Moreover, $\mathrm{ZnO}$ film (deposited by a rf magnetron sputtering) has been demonstrated to be compatible with a CMOS processing [4]. Though $\mathrm{ZnO}$ has relatively low piezoelectric constants, there are many actuator applications whose specifications can be met by $\mathrm{ZnO}$ film. After a careful calculation, we concluded that it is feasible to meet the specifications for an actuated mirror array application with $\mathrm{ZnO}$ film, and have successfully fabricated working arrays (40 $x$ 40) of $\mathrm{ZnO}$-actuated micro-cantilevers.

\section{THEORY AND DESIGN}

For a multi-layer cantilever actuated by a piezoelectric film, the neutral plane is typically designed to be at the bottom plane of the piezoelectric film in order to induce maximum bending stress in the piezoelectric film. For a large deflection, the cantilever should be designed as thin as 
possible. Consequently, we first set the thickness of each layer to its minimum thickness limited by a processing. Then, based on the thickness of each layer, we calculate the thickness of $\mathrm{ZnO}$ to make the neutral plane of the cantilever coincide with the bottom surface of the $\mathrm{ZnO}$ layer. The cross section of our cantilever viewed from the free end is shown in Fig. 3. The requirement that the net axial force on the neutral plane be zero gives the following equation:

$$
P=\sum \Delta P_{i}=\sum \frac{E_{i} y_{i} \Delta A_{i}}{R}=0
$$

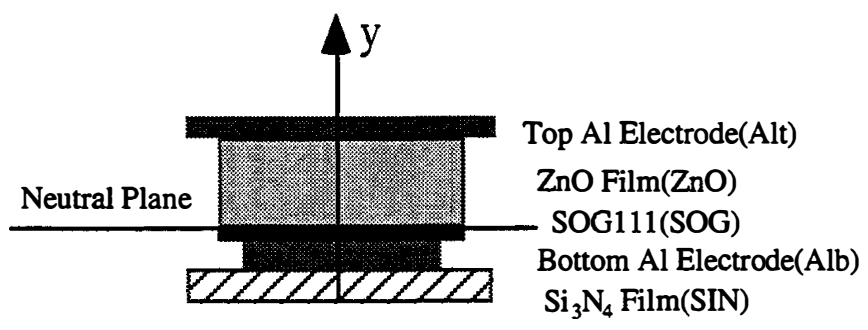

Figure 3. The cross-sectional view of a typical cantilever employed in our mirror array.

where $E_{i}, R$, and $\Delta A_{i}$ are the elastic modulus of a layer $i$, the bending curvature and the cross section area of a layer $i$, respectively. For the multi-layer beam in Fig. 3, we can derive the following equation for the $\mathrm{ZnO}$ thickness $\left(t_{\mathrm{ZnO}}\right)$ :

$$
a t_{Z n O}^{2}+b t_{Z n O}+c=0
$$

where

$$
\begin{aligned}
a= & E_{\mathrm{ZnO}} W_{\mathrm{ZnO}} \\
b= & 2 E_{A l} W_{A l t} t_{A l t} \\
c= & E_{A l} W_{A l t} t_{A l t}^{2}-E_{S O G} W_{S O G} t_{S O G}^{2} \\
& -E_{A l} W_{A l b}\left[\left(t_{A l b}+t_{S O G}\right)^{2}-t_{S O G}^{2}\right]-E_{S i N} W_{S i N} \\
& \times\left[\left(t_{A l b}+t_{S O G}+t_{S i N}\right)^{2}-\left(t_{A l b}+t_{S O G}\right)^{2}\right]
\end{aligned}
$$

where $W_{i}$, and $t_{i}$ are the width and the thickness of a layer $i$, respectively.

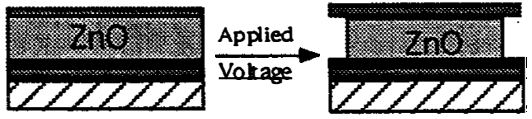

(a)

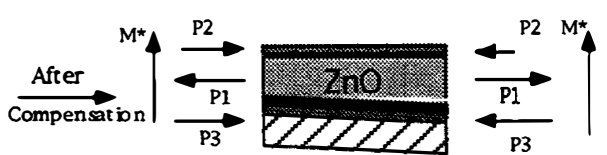

(b)

Figure 4. (a) A segment of the cantilever along the length direction is shown to produce a piezoelectric strain under an applied voltage. The shown strain is for the case where the layers are not attached to each other. (b) The piezoelectric strain for the real case (where all the layers are attached to each other) can be equivalently modeled using forces applied to the layers as shown.
As illustrated in Fig. 4, calculation of the deflection and tip displacement of $\mathrm{ZnO}$-actuated cantilever can be treated as a pure bending with the momentum $M^{*}$ and moment of inertia $I^{*}[5]$. The force densities $\left(P\right.$ in $\left.\mathrm{N} / \mathrm{m}^{2}\right)$ must satisfy the following equations:

$$
\begin{aligned}
& P_{1}=\frac{d_{31} E E_{S i N}\left(S_{2}^{*}+S_{3}^{*}\right)}{S_{1}^{*}+S_{2}^{*}+S_{3}^{*}} \\
& P_{2}=P_{3}=\frac{d_{31} E E_{S i N} S_{1}^{*}}{S_{1}^{*}+S_{2}^{*}+S_{3}^{*}}
\end{aligned}
$$

where $\mathrm{E}$ is an applied electric field across $\mathrm{ZnO}$ film; and $S_{1}^{*}$, $S_{2}^{*}$ and $S_{3}^{*}$ are the effective cross-section areas defined by:

$$
\left\{\begin{array}{c}
S_{1}^{*}=n_{1} W_{A l t} t_{A l t} \\
S_{2}^{*}=n_{2} W_{Z n O} t_{Z n O} \\
S_{3}^{*}=n_{1} W_{A l b} t_{A l b}+n_{3} W_{S O G} t_{S O G}+W_{S i N} t_{S i N}
\end{array}\right.
$$

with $n_{1}=\frac{E_{A l}}{E_{S i N}}, n_{2}=\frac{E_{Z n O}}{E_{S i N}}$ and $n_{2}=\frac{E_{S O G}}{E_{S i N}}$.

Thus, $\mathrm{M}^{*}$ and $\mathrm{I}^{*}$ can be obtained by following equations:

$$
\begin{aligned}
M^{*}= & \frac{P_{1}}{2} n_{2} W_{Z n O} t_{Z n O}^{2}+\frac{P_{2}}{2}\left\{n_{3} W_{S O G} t_{S O G}^{2}\right. \\
& +n_{1} W_{A l b}\left[\left(t_{A l b}+t_{S O G}\right)^{2}-t_{S O G}^{2}\right] \\
& +W_{S i N}\left[\left(t_{A l b}+t_{S i N}+t_{S O G}\right)^{2}-\left(t_{A l b}+t_{S O G}\right)^{2}\right] \\
& \left.-n_{1} W_{A l t}\left[\left(t_{A l t}+t_{Z n O}\right)^{2}-t_{Z n O}^{2}\right]\right\} \\
I^{*}= & \frac{1}{3}\left\{n_{2} W_{Z n O} t_{Z n O}^{3}+n_{1} W_{A l t}\left[\left(t_{A l t}+t_{Z n O}\right)^{3}-t_{Z n O}^{3}\right]\right. \\
+ & n_{3} W_{S O G} t_{S O G}^{3}+n_{1} W_{A l b}\left[\left(t_{S O G}+t_{A l b}\right)^{3}-t_{S O G}^{3}\right] \\
+ & \left.W_{S i N}\left[\left(t_{A l b}+t_{S i N}+t_{S O G}\right)^{3}-\left(t_{S O G}+t_{A l b}\right)^{3}\right]\right\}
\end{aligned}
$$

But the deflection curvature of the cantilever bending $(1 / R)$ is

$$
\frac{1}{R}=\frac{M^{*}}{E_{S i N} I^{*}}
$$

For a cantilever array shown in Fig. 8, the cantilever deflection angle $\theta$ and the tip displacement $\Delta y$ are:

$$
\left\{\begin{array}{c}
\theta=\frac{L_{1}}{R} \\
\Delta y=R(1-\cos \theta)+\left(L-L_{1}\right) \sin \theta
\end{array}\right.
$$

where $L$ and $L_{1}$ are the whole cantilever length and the portion of $L$ that is covered by the $\mathrm{ZnO}$, respectively.

For a cantilever with $L=150 \mu \mathrm{m}$ and $L_{l}=70 \mu \mathrm{m}$, the deflection $\theta$ and the tip displacement $\Delta y$ are calculated to be $0.06 \%$ and $0.12 \mu \mathrm{m} / \mathrm{V}$, respectively.

The fundamental resonant frequency of the cantilever can be estimated by the following equation: 


$$
f_{r}=\frac{1.875^{2}}{2 \pi l^{2}} \sqrt{\left(\frac{E I}{\rho A}\right)_{e f f}}
$$

where $l, E, I, \rho$ and $A$ are the length, the elastic modulus, the moment of inertia, the density and the cross-section area of composite cantilever, respectively. We typically design $f_{r}$ to be about $150 \mathrm{kHz}$, much higher than the operation frequency.

\section{DEVICE FABRICATION}

A $40 \times 40$ array of $\mathrm{ZnO}$ actuated cantilevers (shown in Figs. 6 and 7) is fabricated on a silicon wafer with the fabrication steps illustrated in Fig. 5. The following is a brief description of the 7-mask process. After depositing $\mathrm{Si}_{3} \mathrm{~N}_{4}$ for an electrical isolation, a doped LPCVD polysilicon is deposited and patterned for connecting bottom electrodes. A sacrificial layer of Spin-On-Glass (SOG) is then spincoated and cured in two steps (at $400{ }^{\circ} \mathrm{C}$ for $20 \mathrm{~min}$. and then $850{ }^{\circ} \mathrm{C}$ for one hour). Two types of SOG (SOG311 and SOG511 from Accuglass) are used as sacrificial layers for the thicknesses of $0.5 \mu \mathrm{m}$ and $1 \mu \mathrm{m}$. After patterning the SOG for the anchor area, a cantilever supporting layer of $1300 \AA$ thick low stress LPCVD $\mathrm{Si}_{\mathrm{X}} \mathrm{N}_{\mathrm{y}}$ is deposited. After patterning the silicon nitride with an RIE for an electrical feedthrough window, a $1000 \AA$ thick $\mathrm{Al}$ film is deposited by evaporation for the bottom electrode. Additional layer of $3000 \AA$ thick $\mathrm{Al}$ is deposited for a step coverage. After spin coating a $1800 \AA$ thick SOG (SOG111) for an electrical isolation, $4500 \AA$ thick $\mathrm{ZnO}$ film is sputter-deposited. In a certain HF concentration, the lateral etch of $\mathrm{ZnO}$ by vapor $\mathrm{HF}$ is very slow, and the SOG111 can be patterned by vapor HF using the same photolithography that is used to pattern the $\mathrm{ZnO}$ so that we save one photolithographic step. Finally $800 \AA$ thick $\mathrm{Al}$ is evaporated, and used as the top electrode, the reflective surface and the etch mask during the etch channel formation and removal of the sacrificial layer. The perspective view of the cantilever element is shown in Fig. 8.

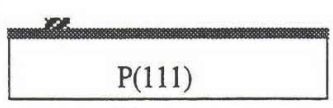

(a)

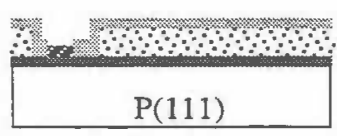

(c)

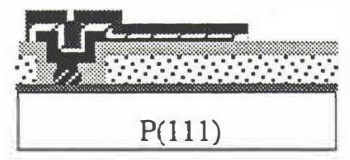

(e)

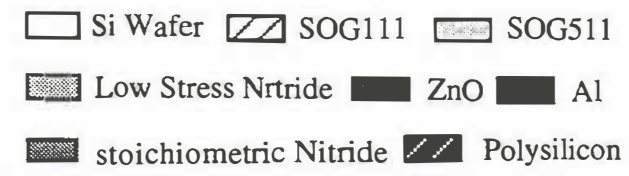

Figure 5. Fabrication process of the cantilever array (cross. sectional views across $A-A^{\prime}$ in Fig.6).

(d)

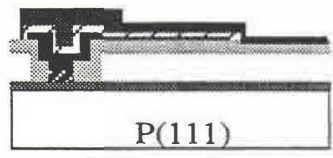

(f)

(b)

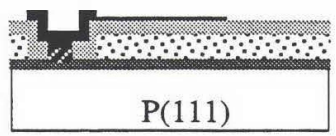

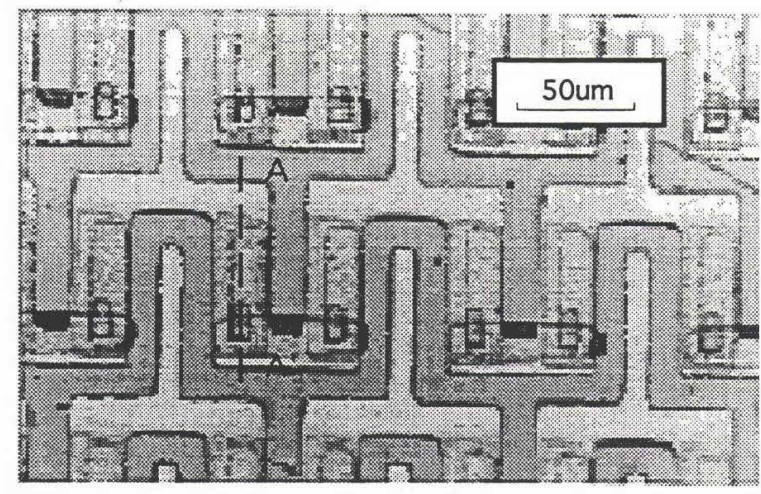

Figure 6. Photograph of close-up top view of the piezoelectrically-actuated cantilever array for a projection display. Each pixel occupies $100 \times 100 \mu \mathrm{m}^{2}$ area.

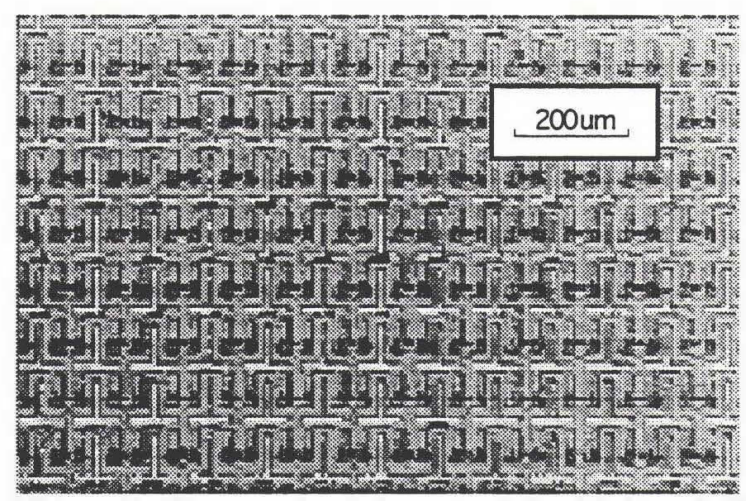

Figure 7. Photograph of the part of the fabricated $40 \times 40$ array of the piezoelectric cantilevers.

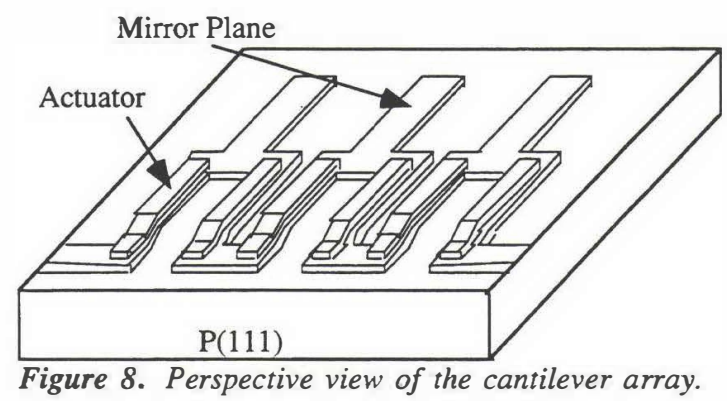

Figure 8. Perspective view of the cantilever array.

Vapor HF is used to release the cantilever. We have discovered that many materials have their unique critical HF concentration below which there is no etching by vapor HF. The critical HF concentration varies depending on a type of a material. As we can see in Fig. 9, Al is rarely etched at a sufficiently diluted vapor $\mathrm{HF}$ (i.e., $75 \mathrm{ml} / \mathrm{min}: 6 \mathrm{ml} / \mathrm{min}$ of N2 : HF), while SOG is still highly etched. Thus, with that HF concentration, bare $800 \AA$ thick $\mathrm{Al}$ can be used as an etch mask during etching SOG to release cantilevers. This discovery has led us to a simpler processing with a higher yield. Specifically the delineation of the top electrode and the etch channel can be performed with one mask: this allows us to save one troublesome step of photoresist strip after the cantilever release. Also we do not have to reserve some margin for protecting $\mathrm{Al}$ during cantilever release, and the surface coverage of the reflective surface can be increased. 


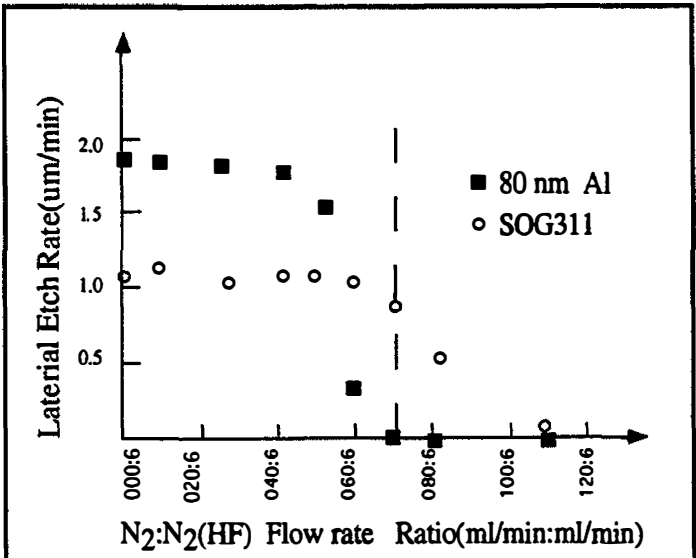

Figure 9. Etch rates of $A l$ and SOG in vapor $H F$.

\section{EXPERIMENTAL RESULTS AND DISCUSSION}

The tip displacement of cantilever by an electrical input is measured with a laser interferometer. In order to distinguish piezoelectric response from thermal response, an electrical input of an unbiased square wave is applied to the cantilever, and a displacement response of a square-wave form is obtained from $0.1 \mathrm{~Hz}$ to as high as $25 \mathrm{kHz}$. The cantilever tips are observed to deflect about $0.19 \mu \mathrm{m} / \mathrm{V}$ vertically in

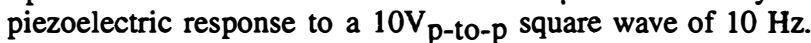
Due to the relative high resistance of each cantilever element (above $100 \mathrm{M} \Omega$ ), the thermal deflection is about 2 or 3 orders of magnitude smaller than the piezoelectrical response according to our theoretical calculations and experimental measurements. Therefore there is no thermal-enhanced deflection in our cantilevers, unlike those reported earlier [6]. A piezoelectric response of cantilever to a $10 \mathrm{~V}_{\text {zero-to-peak }}$ square wave input at $50 \mathrm{~Hz}$ is shown in Figure 10.

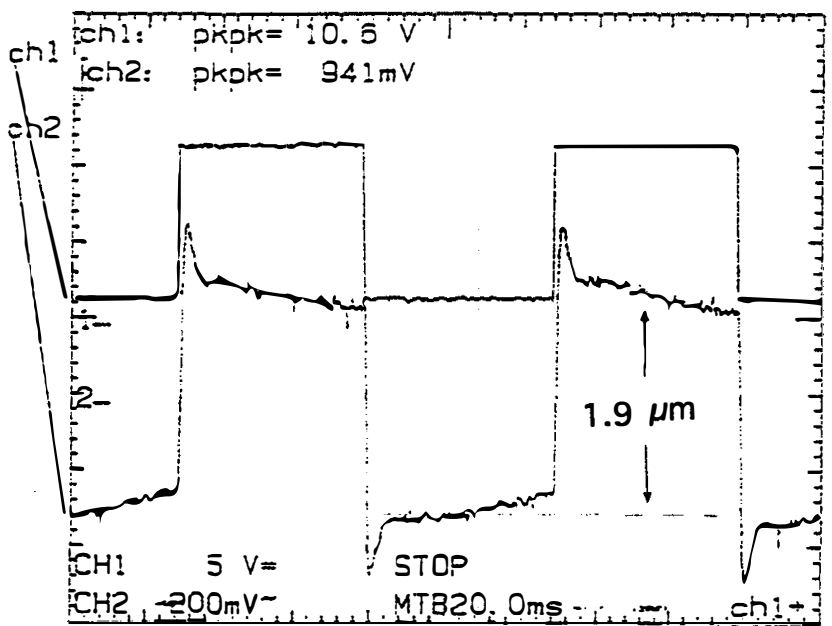

Figure 10. The cantilever-tip displacement (CH2) for a 50

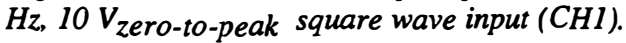

Due to the relative narrow air gap underneath the cantilever, the air damping can reduce the vibrational amplitude of the cantilever significantly. For a cantilever with about $1 \mu \mathrm{m}$ air gap, the vibrational amplitude in the upward direction is observed to be almost twice larger than that in the downward direction. Indeed the frequency response of the cantilevers is dominated by the air gap distance which is determined by the thickness of the sacrificial layer. Typical frequency responses to a square wave with a 5V amplitude are shown in Figs. 11 and 12 for the cantilevers with the air gap of about $0.5 \mu \mathrm{m}$ and $1 \mu \mathrm{m}$, respectively. If the cantilevers are initially deflected about $30^{\circ}$, the roll-off frequency can be as high as $10 \mathrm{kHz}$ as shown in Fig. 13. Thus, the thickness of the sacrificial layer can be a design parameter to control the response time of the cantilever deflection.

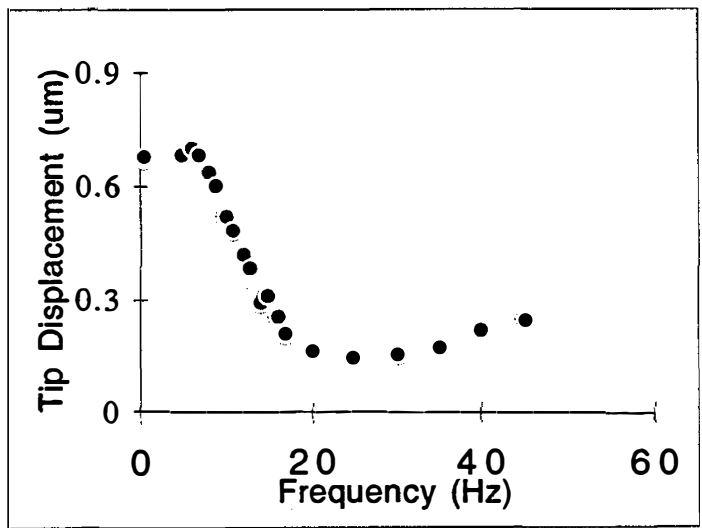

Figure 11. Frequency response of tip displacement of the cantilever with a $0.5 \mu \mathrm{m}$ high air gap.

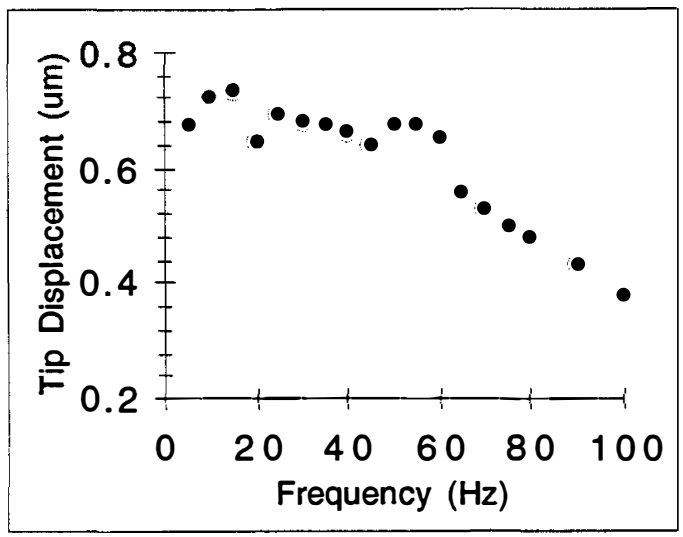

Figure 12. Frequency response of tip displacement of the cantilever with a $1 \mathrm{\mu m}$ high air gap.

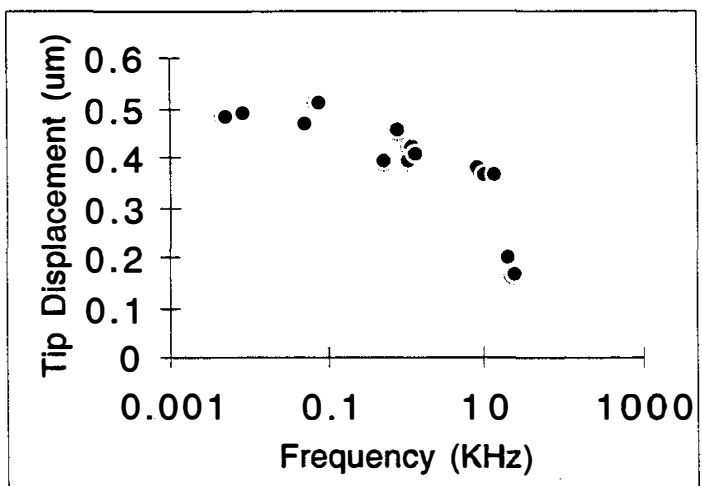

Figure 13. Frequency response of tip displacement of the cantilever that is initially bent up about $30^{\circ}$. 


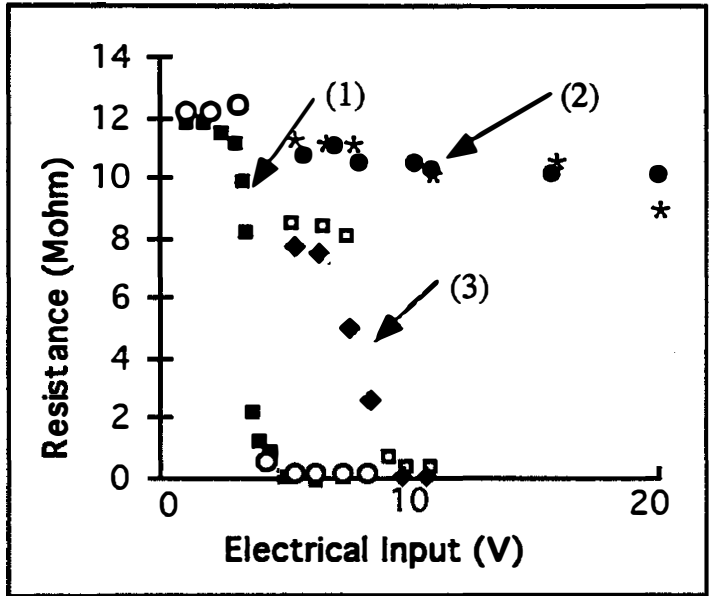

Figure 14. The resistance of $\mathrm{ZnO}$ and thin $\mathrm{SOG}$ on a row of 40 cantilevers as a function of the magnitude of a $8 \mathrm{~Hz}$ square wave.

The cantilever has been observed to deflect linearly in the range of $1-10 \mathrm{~V}$ applied voltage.

The resistivity of $\mathrm{ZnO}$ film in the cantilever has been observed to change due to moisture as confirmed by the data shown in Fig. 14. The data marked with (1) in Fig. 14 are for the devices that have gone through the vapor HF release step, and show sharp drops of the $\mathrm{ZnO}$ resistance when the magnitude of the electrical input ( $8 \mathrm{~Hz}$ square wave) is higher

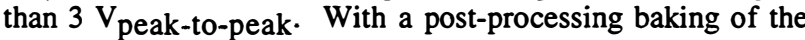
devices at $80^{\circ} \mathrm{C}$ for 2 hours, the resistance drop is non existing even with more than $20 \mathrm{~V}_{\mathrm{p} \text {-to-p }}$ input, as can be seen in the data marked (2) in Fig. 14. The devices exposed to ambient moisture for about one week shows their $\mathrm{ZnO}$ resistance dropping sharply with $8 \mathrm{~V}_{\mathrm{p} \text {-to-p }}$ input (see (3) in Fig. 15). Unless the $\mathrm{ZnO}$ is permanently damaged by too much current, the resistance drops due to moisture can be reversed with baking at $80^{\circ} \mathrm{C}$.

\section{CONCLUSION}

We have demonstrated feasibility of piezoelectric $\mathrm{ZnO}$-actuated cantilevers for a projection display application. A complete fabrication processing for the cantilever arrays has successfully been developed, and used to fabricate working arrays of cantilevers (each array consisting of $40 \mathrm{x}$ 40 cantilevers). Relatively large piezoelectric responses with good linearity have been observed.

\section{ACKNOWLEDGMENT}

The authors wish to thank You Kwang Kim for profiler measurements made at Daewoo Electronics and numerous helps. They also wish to thank Drs. Yun Woo Nam and Jeong Beom Ji of Daewoo Electronics, and Yonghua Song of UH for helpful discussion.

\section{REFERENCE}

1. J.M. Younse, "Mirrors on a chip," IEEE Spectrum, pp. 27 - 31, November 1993.

2. R.B. Apte, F.S.A. Sandejas, W.C. Banyai and D.M. Bloom, "Deformable Grating Light Valves for High
Resolution Displays," Technical Digest of the 1994 SolidState Sensor and Actuator Workshop, Hilton Head Island, SC, June 13 - 16, 1994, Transducer Research Foundation, Cleveland (1994), pp. 1 - 6.

3. G. Um, D. Foley, A. Szilagyi, J. B. Ji, Y. B. Jeon and Y. K. Kim, "S7-6 Recent Advances in Actuated Mirror Array (AMA) Project Development," ASIA DISPLAY (1995), pp. 95 $-98$

4. R.P. Ried, E.S. Kim, D.M. Hong and R.S. Muller, "Piezoelectric Microphone with On-Chip CMOS Circuits," IEEE/ASME Journal of Microelectromechanical Systems, vol. 2, pp. 111-120, September 1993.

5. F.R. Shanley, Strength of Materials, pp. 312-316. New York: McGraw-Hill, 1957.

6. J. G. Smits and W. S. Choi, "Very large Deflection with Quadratic Voltage Dependence of $\mathrm{ZnO}$ on Si3N4 Bimorph," IEEE Transaction on Ultrasonics, Ferroelectrics, Frequencycontrol, vol. 39, no. 2, pp. 302-304, March 1992. 